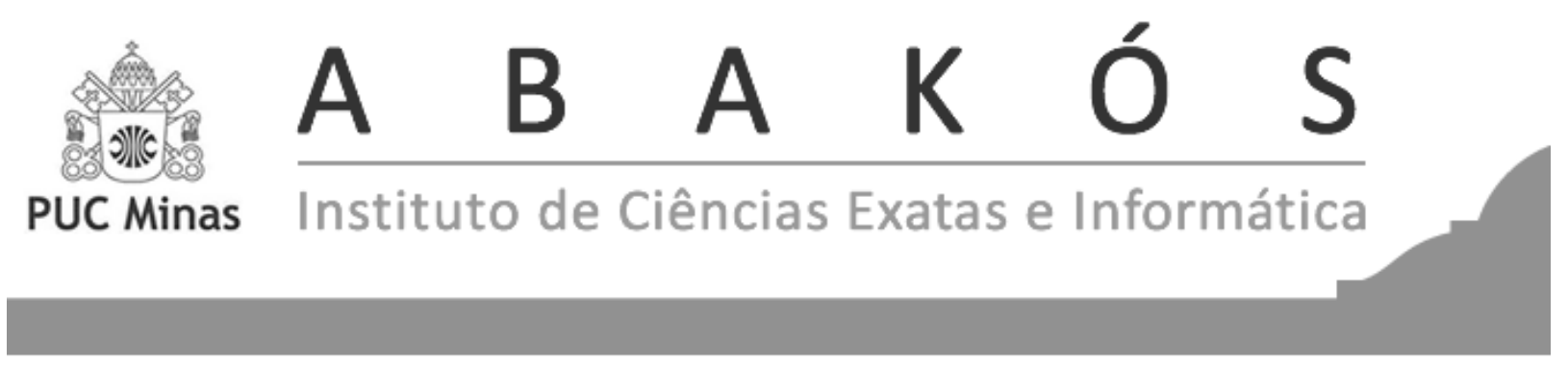

\title{
Sala de Aula Invertida: Cenários Atuais na Formação de Engenheiros*
}

Flipped Classroom: Current Scenarios in Engineering Education

\author{
Caroline Subirá Pereira ${ }^{1}$ \\ Cristiane de Fatima Budek Dias ${ }^{2}$ \\ Marcos Mincov Tenório ${ }^{3}$ \\ Guataçara dos Santos Junior ${ }^{4}$
}

\begin{abstract}
Resumo
A Sala de Aula Invertida é uma proposta de ensino híbrido que vem sendo muito discutida e aplicada na formação de engenheiros nos cursos do Ensino Superior. Este artigo visa investigar a viabilidade da metodologia Sala de Aula Invertida, aplicada na formação de engenheiros, a partir das publicações científicas que trabalham com esta temática. Para atingir esse objetivo seguiu-se a metodologia de revisão sistemática Methodi Ordinatio, a qual faz uso de passos sistêmicos que possibilitam a ordenação dos artigos por relevância, considerando três importantes características: o número de citações, o fator de impacto e o ano da publicação. A busca pelos artigos foi realizada nas bases: Science Direct, Scielo, Scopus e Web of Science, no ano de 2018. Foram encontrados 325 artigos e a análise mais detalhada se deu sobre os 15 primeiros trabalhos classificados pela ordem de relevância dada pela metodologia utilizada. Os resultados apontaram para ausência de material teórico que direcione a prática docente a partir do modelo pedagógico da Sala de Aula Invertida, para a escassez da abordagem do tema na formação docente e para dificuldades docentes para a prática. Em contrapartida, aponta para os benefícios do modelo visando a formação de engenheiros com eficácia.
\end{abstract}

Palavras-chave: Sala de aula invertida. Ensino superior. Formação de Engenheiros. Metodologias de ensino.

\footnotetext{
* Submetido em 16/12/2019 - aceito em 12/04/2021.

${ }^{1}$ Doutoranda do Programa de Pós-Graduação em Ensino de Ciência e Tecnologia da Universidade Tecnológica Federal do Paraná. UTFPR, Brasil - carolinepereira@ alunos.utfpr.edu.br.

${ }^{2}$ Doutoranda do Programa de Pós-Graduação em Ensino de Ciência e Tecnologia da Universidade Tecnológica Federal do Paraná. UTFPR, Brasil - cristianed@alunos.utfpr.edu.br.

${ }^{3}$ Professor Doutor do Departamento Acadêmico de Informática da Universidade Tecnológica Federal do Paraná. UTFPR, Brasil - marcostenorio@utfpr.edu.br

${ }^{4}$ Professor Doutor do Programa de Programa de Pós-Graduação em Ensino de Ciência e Tecnologia da Universidade Tecnológica Federal do Paraná. UTFPR, Brasil - guata@utfpr.edu.br
} 


\begin{abstract}
The flipped classroom is a hybrid teaching proposal that has been discussed and applied in engineering education on higher education. This paper aims to investigate the pedagogical model of Inverted Classroom, applied in the training of engineers, from the scientific publications that discuss this theme. We used the methodology of systematic review called Methodi Ordinatio which makes use of systemic steps that allow the ordering of articles by relevance, considering three important characteristics: the number of citations, the impact factor and the year of publication. The search for articles was carried out at the bases: Science Direct, Scielo, Scopus and Web of Science. From the 325 articles found, a more detailed analysis was carried out on the first 15 papers classified by order of relevance. The results pointed to the absence of theoretical material to direct the teaching practice from the pedagogical model of the Inverted Classroom, the scarcity of the approach of the subject in the teacher training and teaching difficulties for the practice. However, in contrast, it points to the benefits of the model in order to train engineers effectively.
\end{abstract}

Keywords: Inverted classroom. Higher education. Engineering education. Teaching methodologies. 


\section{INTRODUÇÃO}

Diante do atual nível tecnológico que as indústrias se encontram, é natural que o mercado de trabalho amplie as exigências quanto a formação dos engenheiros. De um modo geral, os cursos de formação de engenheiros visam formar um profissional apto para apresentar soluções de acordo com sua área específica de formação, com habilidades e competências compatíveis com o avanço tecnológico da sociedade e das indústrias. Para atender esses objetivos tem-se investido em Metodologias Ativas de ensino.

As Metodologias Ativas podem ser compreendidas como propostas de ensino com foco no aluno. Elas são caminhos que proporcionam o desenvolvimento da autonomia, desenvolvem uma formação crítica, despertam a curiosidade e capacitam para tomada de decisões (BORGES; ALENCAR, 2014). Como exemplos de Metodologias Ativas citam-se: Aprendizagem Baseada em Problemas, Aprendizagem Orientada por Projetos, Sala de Aula Invertida, entre outras metodologias que são centradas no aluno.

Para este estudo peculiariza-se a metodologia da Sala de Aula Invertida aplicada a formação de engenheiros. Nessa metodologia as instruções e os conteúdos são enviados ao aluno antes da aula. O aluno estuda online, "usando as $\mathrm{TDIC}^{5}$, mais especificamente, os ambientes virtuais de aprendizagem. A sala de aula torna-se o lugar de trabalhar os conteúdos já estudados, realizando atividades práticas como resolução de problemas e projetos, discussão em grupo e laboratórios" (VALENTE, 2018, p. 27).

Este artigo tem a finalidade de responder a seguinte pergunta: Qual a viabilidade da metodologia Sala de Aula Invertida para a formação de engenheiros? E visando a resposta desta pergunta, este artigo tem por objetivo investigar a viabilidade da metodologia Sala de Aula Invertida, aplicada na formação de engenheiros, a partir das publicações científicas que abordam esse temática.

Para atingir esse objetivo, e responder a pergunta de partida, seguiu-se a metodologia de revisão sistemática Methodi Ordinatio, elaborada por Pagani, Kovaleski e Resende (2015), a qual faz uso de passos sistêmicos que possibilitam a ordenação dos artigos científicos por relevância, considerando três importantes características: o número de citações, o fator de impacto e o ano da publicação. A busca pelos artigos foi realizada nas bases: Science Direct, Scielo, Scopus e Web of Science, considerando que são bases pertinentes na área de Ensino.

Com uma abordagem qualitativa, posterior ao levantamento dos artigos, construiu-se uma análise interpretativa dos trabalhos com olhar para os resultados da aplicação do modelo pedagógico na formação de engenheiros. E com uma abordagem quantitativa, verificou-se a evolução do tema e ilustrou-se um grafo, explicitando a teia dos autores das produções. As próximas seções esclarecem sobre as etapas do método escolhido para a revisão sistemática, explanam sobre a análise e apresentam os resultados.

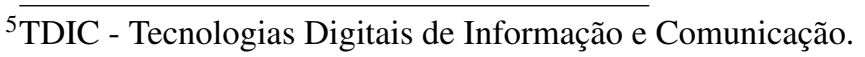




\section{PROCEDIMENTOS METODOLÓGICOS}

A metodologia Methodi Ordinatio foi escolhida para realização da revisão sistemática neste trabalho uma vez que permite organizar as publicações científicas por ordem de relevância. A classificação dos artigos leva em consideração o número de citações, o fator de impacto da revista e o ano da publicação, resultando em um valor denominado InOrdinatio. Assim, o valor InOrdinatio resultante reflete a relevância do artigo analisado.

Para determinação deste índice faz-se uso da Equação 1.

$$
\text { InOrdinatio }=(F i / 1000)+a *[10-(\text { AnoPesq }- \text { AnoPub })]+C i
$$

que Fi representa o fator de impacto do periódico, $\alpha$ um valor atribuído pelo próprio pesquisador (1 a 10) para refletir a importância do ano de publicação dentro da equação. Quanto mais elevado o valor de $\alpha$, maior o peso referente ao ano da publicação (pesquisa mais recente). Para este estudo adotou-se $\alpha=10$, levando-se em conta a pretensão de um estudo do contexto atual. E Ci é o número de citações que o artigo possui (PAGANI; KOVALESKI; RESENDE, 2015).

A Methodi Ordinatio contempla nove passos (PAGANI; KOVALESKI; RESENDE, 2015). O primeiro se refere à definição da intenção da pesquisa, que neste artigo é a de investigar sobre a metodologia Sala de Aula Invertida aplicada na formação de engenheiros a partir das publicações científicas que trabalham com esta temática. O segundo passo consiste em uma pesquisa preliminar nas bases de dados, na intenção de auxiliar o pesquisador a delimitar as palavras-chave que serão aplicadas na busca definitiva.

O terceiro passo é a escolha das palavras-chave. Para este artigo, após os testes preliminares e com o uso de operadores booleanos para refinamento dos dados, determinou-se a combinação de palavras-chave: "flipped classroom" AND "higher education" AND "engineer* education". No passo seguinte efetiva-se a busca definitiva dos artigos, efetuada nas bases: Science Direct, Scielo, Scopus e Web of Science, consideradas pertinentes para a área de estudo desta pesquisa. A busca foi realizada em setembro de 2018 e a Tabela 1 exibe a quantidade de artigos encontrados em cada uma das bases consultadas.

Tabela 1 - Resultado da busca

\begin{tabular}{ccccc}
\hline Science Direct & Scielo & Scopus & Web of Science & TOTAL \\
\hline 170 & 1 & 151 & 3 & 325 \\
\hline
\end{tabular}

Fonte: Dados da pesquisa.

O quinto passo consiste no processo de filtragem, visando apenas os artigos que realmente estão alinhados à intenção da pesquisa. O primeiro critério de filtragem adotado para esta revisão sistemática foi a exclusão dos artigos duplicados. O processo de verificação de duplicidade foi facilitado pelo software Mendeley (ferramenta usada para arquivar os dados baixados das bases). Com esse critério, cinco artigos foram eliminados. O segundo critério de filtragem foi a exclusão de artigos com títulos e resumos desalinhados ao tema, feito a partir da leitura do 
título e resumo. Ao final da filtragem o número de artigos final foi de 111.

Após a filtragem, inicia-se o sexto passo, que consiste na identificação do número de citações de cada artigo e o fator de impacto dos periódicos. O número de citações foi identificado com o auxílio do Google Acadêmico, e o fator de impacto consultado por meio do endereço: https://www.scopus.com/sources. A partir dessas informações, efetuou-se o sétimo passo, que consiste na aplicação da Equação 1 e ordenação do InOrdinatio do maior para o menor. Por fim, o oitavo e nono passos que, respectivamente, referem-se ao download dos artigos e leitura sistemática para posterior análise. A leitura sistemática foi empregada sobre os 15 primeiros trabalhos que correspondem às publicações mais relevantes. O próximo tópico apresenta esses dados e resultados.

\section{RESULTADOS}

A explanação dos resultados está organizada em dois subtópicos, o primeiro com abrangência qualitativa e o segundo com aspecto quantitativo. No Quadro 1 estão expostos os títulos, autores e ano de publicação dos 15 primeiros artigos ordenados em ordem decrescente no InOrdinatio, seguido dos códigos que serão adotados no decorrer da análise. Utiliza-se o código (Cod.) representado pela letra A (artigo), seguido de ponto (.) e de um número inteiro $(1,2$, $3 . .$.$) que representa a posição em que o trabalho foi classificado.$ 


\section{Quadro 1 - Quinze primeiros artigos}

\begin{tabular}{|c|c|c|c|}
\hline Cod. & Título do Artigo & Autores & Ano \\
\hline A. 1 & $\begin{array}{l}\text { Motivation and cognitive load in the flipped classroom: definition, } \\
\text { rationale and a call for research }\end{array}$ & Abeysekera e Dawson & 2015 \\
\hline A. 2 & $\begin{array}{l}\text { Comparing the Effectiveness of an Inverted Classroom to a Traditional } \\
\text { Classroom in an Upper-Division Engineering Course }\end{array}$ & $\begin{array}{l}\text { Mason, Shuman e } \\
\text { Cook. }\end{array}$ & 2013 \\
\hline A. 3 & $\begin{array}{l}\text { The experience of three flipped classrooms in an urban university: an } \\
\text { exploration of design principles }\end{array}$ & Kim et al. & 2014 \\
\hline A. 4 & $\begin{array}{l}\text { It's not about seat time: Blending, flipping, and efficiency in active } \\
\text { learning classrooms }\end{array}$ & Baepler, Walker e Driessen & 2014 \\
\hline A. 5 & $\begin{array}{l}\text { The Evolution of a Flipped Classroom: Evidence-Based } \\
\text { Recommendations }\end{array}$ & $\begin{array}{l}\text { Velegol, Zappe e } \\
\text { Mahoney }\end{array}$ & 2015 \\
\hline A. 6 & $\begin{array}{l}\text { A systematic review of research on the flipped learning method in } \\
\text { engineering education }\end{array}$ & $\begin{array}{l}\text { Karabulut-Ilgu, } \\
\text { Cherrez e Jahren. }\end{array}$ & 2018 \\
\hline A. 7 & $\begin{array}{l}\text { Exploring students' learning attitude and achievement in flipped } \\
\text { learning supported computer aided design curriculum: A study in high } \\
\text { school engineering education }\end{array}$ & Chao, Chen e Chuang & 2015 \\
\hline A. 8 & $\begin{array}{l}\text { Exploring the role of e-learning readiness on student satisfaction and } \\
\text { motivation in flipped classroom }\end{array}$ & Yilmaz & 2017 \\
\hline A. 9 & $\begin{array}{l}\text { Implementing flipped classroom using digital media: A comparison of } \\
\text { two demographically different groups perceptions }\end{array}$ & Sohrabi e Iraj & 2016 \\
\hline A. 10 & Learning analytics to unveil learning strategies in a flipped classroom & $\begin{array}{l}\text { Jovanović, Gašević, } \\
\text { Dawson, Pardo, } \\
\text { Mirriahi }\end{array}$ & 2017 \\
\hline A. 11 & $\begin{array}{l}\text { Use of the flipped classroom instructional model in higher education: } \\
\text { instructors' perspectives }\end{array}$ & $\begin{array}{l}\text { Long, Cummins e } \\
\text { Waugh }\end{array}$ & 2017 \\
\hline A. 12 & $\begin{array}{l}\text { Development of an instructional design model for flipped } \\
\text { learning in higher education }\end{array}$ & Lee, Lim e Kim & 2017 \\
\hline A. 13 & Exploring Flipped Classroom Instruction in Calculus III & $\begin{array}{l}\text { Wasserman, Quint, } \\
\text { Norris e Carr }\end{array}$ & 2017 \\
\hline A. 14 & $\begin{array}{l}\text { Inverted learning environments with technology, innovation and } \\
\text { flexibility: Student experiences and meanings }\end{array}$ & $\begin{array}{l}\text { Ramírez-Montoya e } \\
\text { Hernández }\end{array}$ & 2016 \\
\hline A. 15 & $\begin{array}{l}\text { The flipped classroom in further education: literature review and case } \\
\text { study }\end{array}$ & Little & 2015 \\
\hline
\end{tabular}

\section{Fonte: Dados da pesquisa.}

Os subtópicos seguintes descrevem as análises e explicitam os resultados conforme interpretação dos autores desta pesquisa.

\subsection{Análise qualitativa dos dados}

O artigo A.1 não coloca discussões específicas para a formação de engenheiros, mas possui o InOrdinatio mais elevado dentro da classificação e traz considerações importantes sobre a Sala de Aula Invertida como modelo pedagógico, por isso foi mantido dentro do grupo a ser analisado.

O estudo foi realizado na Austrália, e coloca a ideia da Sala de Aula Invertida como 
proposta intencional de substituir a aula tradicional por atividades ativas para os alunos. $\mathrm{O}$ conteúdo teórico, que geralmente é abordado na sala de aula tradicional, é transferido e disponibilizado para o aluno fora da sala de aula, em um momento anterior, dando o sentido de inversão.

Dentre os artigos incluídos no Quadro 1, também se observou estudos de aplicação do modelo pedagógico Sala de Aula Invertida e análises dos resultados quanto ao ensino e aprendizagem na formação de engenheiros. Em A.2, Mason, Shuman e Cook (2013) realizam uma análise comparativa do ensino tradicional com o ensino invertido.

O estudo publicado em A.2, realizado nos Estados Unidos da América, mostrou que a Sala de Aula Invertida proporciona mais conhecimento aos alunos, pois os autores concluíram que, com a inversão da sala de aula, mais conteúdo é alcançado no decorrer do curso. Os alunos participantes da pesquisa, mostraram melhores resultados de aprendizagem. Em outro estudo realizado nos Estados Unidos, o A.4, também se constata os aspectos positivos para o ensino e aprendizagem na formação de engenheiros com essa metodologia.

Já os autores de A.3, que se refere a mais um estudo realizado nos Estados Unidos, preocupam-se com as dificuldades dos docentes em utilizar a metodologia da Sala de Aula Invertida. Criar ambientes de aprendizado centrados no aluno exigem aplicação de estratégias que possibilitem aprendizagem ativa como: pequenos grupos de resolução de problemas, discussões construtivas, exposições e apresentações e avaliação por pares. E isso pode trazer alguns obstáculos para o docente.

Segundo Kim, Kim, Khera e Getman (2014) os docentes nem sempre estão preparados para implementar estas estratégias de aprendizagem ativa. Assim confrontam-se os resultados satisfatórios de A.2 e A.4 com as dificuldades apontadas em A.3, referentes à formação docente, direcionando para uma possível lacuna para a efetivação da Sala de Aula Invertida como metodologia para a formação de engenheiros.

Em A.5 os autores colocam que as dificuldades para o ensino de engenharia se relacionam entre o desafio de ministrar muitas aulas teóricas e proporcionar momentos de interação ativa com o conteúdo teórico, com o objetivo de melhor aprendê-lo por meio de aplicações (VELEGOL; ZAPPE; MAHONEY, 2015, p. 27).

Velegol, Zappe e Mahoney (2015) (A.5), concluíram em seu estudo que os alunos do curso de Engenharia da Pennsylvania, Estados Unidos, preferem flexibilidade para aprender o conteúdo teórico e mais momentos de interação em sala de aula. Isso mostra que a Sala de Aula Invertida atende as necessidades dos alunos, pois disponibiliza o conteúdo teórico antes das aulas, momento em que o próprio aluno tem autonomia para se organizar e estudar.

Já o artigo A.6 aproxima-se da presente pesquisa, pois condiz com uma revisão de literatura a partir de artigos que abordam a mesma temática. Karabulut-Ilgu, Jaramillo Cherrez e Jahren (2018), autores de A.6, realizaram uma busca por artigos em várias bases publicados entre 2000 e 2015 encontrando 62 artigos. As diferenças entre o presente artigo e os resultados de A.6 se explicitam pelo fato dos autores de A.6 não esclarecerem sobre quais foram as bases consideradas para a busca que resultou em um número relativamente pequeno comparado ao 
resultado desta atual busca. A limitação de tempo também difere do presente artigo, visto que, na revisão sistemática realizada aqui não há limitação de ano.

Em A.6 os resultados apontam para uma popularidade do modelo pedagógico Sala de Aula Invertida no Ensino Superior, especificamente para a formação de engenheiros. A revisão de literatura de A.6 também mostrou que há uma escassez de relatos de referenciais teóricos para orientar docentes quanto ao desenvolvimento e planejamento de um curso nos moldes da Sala de Invertida.

A.7 resgata os pontos positivos da eficácia do modelo pedagógico Sala de Aula Invertida apontados por A.2 e A.4, mas 'reinventa' e completa o modelo, pois mostra os benefícios de um estudo desenvolvido na República da China, que aliou os preceitos da Sala de Aula Invertida com a Aprendizagem Colaborativa Baseada em Problemas (do inglês Collaborative Problem Based Learning - CPBL). Os autores de A.7 exploram os benefícios, em relação ao desempenho, atitude para aprender e aprendizagem dos estudantes em práticas de Sala de Aula Invertida com suporte na metodologia CPBL.

Os resultados mostrados em A.7 revelam que a CPBL foi facilitada com a abordagem da Sala de Aula Invertida, também eficaz para a atitude de aprendizagem dos estudantes, assim como para o desenvolvimento de uma aprendizagem colaborativa. Os autores afirmam que tais fatos trazem mudanças na cultura de aprendizagem, uma vez que os alunos demonstraram atitudes positivas de aprendizagem, aprenderam a compartilhar e discutir com os colegas, se envolveram ativamente na construção do conhecimento e na autoavaliação.

Chao, Chen e Chuang (2015), (A.7), mencionam que os alunos assentiram que a metodologia adotada foi oportuna para a aprendizagem por meio da resolução de um problema prático de construção de uma ponte, abordando a tecnologia de construção, o design, a estrutura, os materiais e o projeto usando software, sendo que alguns estudantes apontaram que, ter o contato com vídeos sobre os conteúdos em momentos anteriores às aulas, facilitou a compreensão e, consequentemente, a discussão nos momentos de aula, oportunizando a resolução do problema apresentado pelo professor em um curto espaço de tempo. Além disso, houve a ampliação do conhecimento e do pensamento, pois puderam ter acesso a diversas ideias propostas por seus colegas.

Todavia, os apontamentos de A.3 e A.6, quanto a dificuldade dos docentes em implementar estratégias de aprendizagem ativa e a ausência de suporte teórico para orientar a implementação destas estratégias, oportunizam a combinação de outros modelos pedagógicos, ou seja, apontam para práticas em que a combinação de estratégias pode ser um caminho viável para a implementação efetiva da Sala de Aula Invertida. A.7 é um exemplo dessa situação, que combina Sala de Aula Invertida com Aprendizagem Colaborativa Baseada em Problemas. Entretanto, apesar dos resultados positivos da aplicação da combinação, não se esclarecem informações teóricas e não há preocupação com a formação do docente para futuras aplicações, como nos artigos A.3 e A.6.

A.8 explora a prontidão dos alunos para a aprendizagem online (e-learning). O estudo foi realizado em uma instituição de Ensino Superior na Turquia. O autor Yilmaz (2017) afirma 
que um dos grandes desafios na implementação da metodologia da Sala de Aula Invertida está relacionado à prontidão $e$-learning, que afeta a satisfação e a motivação dos estudantes, desse modo, analisa como a prontidão para essa aprendizagem afeta tais aspectos.

Para chegar aos resultados, o autor de A.8 aplicou três instrumentos de autorrelato: Escala de Prontidão e-learning, Escala de Satisfação e Questionário de Estratégias de Motivacionais para Aprendizagem de Pintrich e Groot (1990). Com isso, concluiu que a prontidão e-learning precede, significativamente, a satisfação e a motivação em abordagens com uso da metodologia da Sala de Aula Invertida.

Yilmaz (2017) (A.8) afirma que para o desempenho ativo que se espera dos estudantes na realização das atividades propostas e a satisfação e motivação para essa tarefa, é fundamental que os alunos estejam munidos de conhecimento prévio sobre o conteúdo, por isso, o nível de prontidão para a aprendizagem online é imprescindível para a eficácia da Sala de Aula Invertida. Para tanto, sugere que o estudante tenha habilidades como: auto-eficácia no uso do computador, da internet e na comunicação. É necessário, ainda, habilidades para a aprendizagem autodirigida, controle do aprendiz e motivação para a aprendizagem online.

Deste modo Yilmaz (2017) alerta que para um melhor aproveitamento do modelo pedagógico Sala de Aula Invertida, para aumentar os níveis de prontidão para a aprendizagem online dos alunos, se faz necessário um processo anterior, que auxilie no desenvolvimento das habilidades acima mencionadas. Isso pressupõe que se deve ter atenção na introdução dessa metodologia, pois é importante que os estudantes estejam motivados e sintam satisfação para uma aprendizagem mais efetiva.

No artigo A.9 os autores fazem uma análise, a partir de dois grupos de alunos demograficamente diferentes, e, de um modo geral observou-se que os alunos estão procurando por novas 'salas de aula'. Assim observa-se que a busca por novos modelos pedagógicos não é uma ação exclusiva dos docentes, mas há casos em que é de interesse dos próprios alunos a procura por metodologias ativas.

A.10 analisa as estratégias de aprendizagem de estudantes australianos. Os dados para análise são oriundos das atividades preparatórias propostas aos alunos, as quais deveriam ser concluídas antes da aula presencial, ou seja, a etapa inicial da Sala de Aula Invertida.

Por meio da análise realizada os autores de A.10 notaram que os estudantes mais ativos na regulação da sua aprendizagem foram os que apresentaram maior desempenho na disciplina. Houve, entretanto, indicativos da adoção de estratégias de aprendizagem distintas pelos alunos, prevalecendo estratégias mais mecânicas e contrárias a comportamentos ativos. Daí tem-se a importância de preparar os alunos para o modelo pedagógico a ser adotado conforme apontado em A.8.

A.11 tem como foco de estudo os professores, analisando suas experiências e perspectivas com a Sala de Aula Invertida. Para essa análise, os autores realizaram entrevistas com professores que utilizaram esse modelo pedagógico em suas aulas. O estudo foi realizado nos Estados Unidos, com professores do Ensino Superior de diferentes disciplinas.

Long, Cummins e Waugh (2017), autores de A.11, revelam que todos tendem para a 
definição de que a Sala de Aula Invertida é aquela em que os estudantes têm contato com os conteúdos antes da aula, sendo um estudo autodirigido. Mas mesmo com definições semelhantes, os professores dão focos diferenciados em seus relatos, uns enfatizam a mudança na função docente, enquanto outros nas habilidades e na aprendizagem ativa dos estudantes.

Esse resultado pode ter relação com a falta de material teórico e preparação docente que A.3 e A.6 apontam, pois explicita que não há um caminho bem definido quanto a Sala de Aula Invertida enquanto modelo pedagógico. Sobre as formas de utilização da metodologia da Sala de Aula Invertida, os professores da pesquisa realizada em A.11 indicam objetivos comuns de uso, como transformar a aprendizagem passiva em aprendizagem ativa e oportunizar mais tempo e espaço para os alunos aplicarem seus conhecimentos durante a aula.

Em relação aos benefícios percebidos pelos participantes do estudo, Long, Cummins e Waugh (2017) mencionam a boa aceitação e a motivação dos estudantes no modelo de aula invertida; a melhoria no aproveitamento do tempo em sala de aula, com a aplicação de exercícios práticos, o que beneficiou a aprendizagem e as habilidades de pensamento dos estudantes; o suporte imediato às dúvidas dos alunos durante a realização de exercícios em aula e a interatividade entre professores/estudantes e estudantes/estudantes.

Os desafios apontados pelos professores, da pesquisa de A.11, dizem respeito à preparação dos estudantes antes da aula, relacionando-se novamente com os resultados de A.8. A dificuldade na leitura de materiais e exercícios disponibilizados para serem realizados antes do momento presencial em aula; a não aceitação de alguns estudantes no modelo invertido, pois alguns podem sentir-se mais confortáveis com aulas tradicionais; a falta de colaboração entre os estudantes nas reflexões e revisões e o tempo dispensado pelos docentes na preparação e execução das aulas invertidas.

A aplicação eficiente da Sala de Aula Invertida, segundo os pesquisados em A.11, se dá a partir de quatro componentes principais: garantir a preparação dos alunos antes da aula; organizar boas instruções; organizar materiais e atividades com base no retorno dos alunos, e fornecer suporte apropriado e imediato durante a aula. Para garantir que os alunos estivessem preparados para a aula, os professores utilizaram mecanismos como: apresentação de anotações dos vídeos e materiais disponibilizados, realização de exercícios e aplicação de conceitos (notas).

Para organizar boas instruções os professores sugerem a atenção a objetivos claros e consistentes com o que se deseja dos alunos; a organização de cronogramas de acesso aos materiais, estudo e avaliação. Isso revela a importância de um bom planejamento, sendo que este deve ser claro, conciso e flexível para que se possa atender às necessidades dos alunos, de acordo com o retorno observado nas aulas. Tal aspecto é apontado pelos professores, que mencionam que conhecer necessidades e ter um feedback dos estudantes é fundamental para a eficácia da Sala de Aula Invertida. Para ter esse conhecimento sobre o aluno, os professores pesquisados buscaram se colocar como alunos, ter o feedback de alunos mais silenciosos e pensar nos conhecimentos que os alunos já possuem.

O fornecimento de suporte apropriado e imediato durante a aula é o último componente 
apontado pelos professores como sendo essencial para a eficiência da aula invertida. Nesse componente apontam para a colocação de desafios aos alunos, para a estimulação do pensamento do estudante na resolução dos problemas para que sejam ativos e busquem soluções por si mesmos. Desse modo, entende-se que o suporte apropriado não significa dar respostas prontas às dúvidas dos alunos, mas instigar o pensamento e a reflexão sobre o problema.

Outro ponto ressaltado em A.11 é a colaboração entre pares, ou seja, a interação com colegas de profissão, que possam dar suporte, compartilhar experiências e conhecimentos. $\mathrm{O}$ que se percebe no texto de A.11, é que isso foi um dos pontos fortes relatados pelos professores pesquisados. Tal aspecto contribuiu para a eficiência na aplicação do modelo invertido de aula.

O estudo realizado em A.11 se deu com professores que passaram por curso de formação para a aplicação da metodologia de Sala de Aula Invertida e seus resultados mostram benefícios e estratégias utilizadas pelos professores na busca da superação dos desafios. Esse estudo reafirma a relevância da formação docente para a eficiência na implementação da metodologia. Volta-se aqui para a lacuna da formação apontada em A.3, que menciona as dificuldades dos professores para as boas práticas da Sala de Aula Invertida.

De um modo geral Long, Cummins e Waugh (2017) acreditam que o grande desafio e, possivelmente, a lacuna que precisa ser preenchida, está na preparação dos alunos antes da aula, ou seja, na garantia de que os estudantes realizem as tarefas direcionadas para que a aula seja o momento de discussão e reflexão sobre o conteúdo. Essa motivação dos alunos antes da aula é o objeto de pesquisa de A.8, que revela que a prontidão para a aprendizagem online é elemento fundamental para que a Sala de Aula Invertida traga benefícios para a aprendizagem.

A.12 apresenta um modelo de projeto de aula invertida para o Ensino Superior. O modelo foi desenvolvido e validado por meio de testes de usabilidade, revisão de especialistas e avaliação de campo (aplicação em uma sala de aula com estudantes sul-coreanos).

O modelo de Lee, Lim e Kim (2017), autores de A.12, apresenta uma combinação de atividades online, face a face, individuais e colaborativas, que buscam articular coerentemente experiências de aprendizagem, atendendo a necessidades e contextos específicos. No modelo há um passo a passo para a elaboração de um projeto de Sala de Aula Invertida, atendendo a questões macro e micro, específicas da disciplina e dos conteúdos.

$\mathrm{Na}$ validação em campo, os autores de A.12 mencionam satisfação dos alunos com o modelo implementado. Além disso, apontam para uma melhoria da aprendizagem na disciplina em que foi aplicado. Uma questão que fica evidente no modelo apresentado por Lee, Lim e Kim (2017) é o tempo de preparo dos materiais e atividades, pois agrega a gravação, edição e disponibilização de vídeos, bem como a elaboração de atividades e questionários para os estudantes. Esse ponto corrobora com o desafio apontado pelos professores participantes da pesquisa descrita em A.11.

A.13 traz um estudo realizado nos Estados Unidos que compara os efeitos da Sala de Aula Invertida na disciplina de Cálculo III sobre desempenho e a percepção dos alunos em relação a uma classe de aula tradicional. Os dados coletados em dois semestres indicam que o desempenho foi semelhante entre os dois grupos de alunos. Em relação à percepção dos 
estudantes, aqueles submetidos a práticas de aula invertida, mencionam um aumento da comunicação nas aulas, o que mostra mais um ponto satisfatório como resultado: o desenvolvimento ativo do aluno.

No México houve um investimento inovador e tecnológico ligado diretamente ao estudante universitário, e o A.14 faz a análise desse investimento que se baseou no modelo de Sala de Aula Invertida ${ }^{6}$. Os resultados mostraram que os estudantes universitários modificaram seus horários e locais de estudo; notou-se que a aprendizagem ativa está relacionada aos significados dos estudantes, e percebeu-se que eles encontraram uma fonte de aprendizado não apenas na figura do professor, mas também em seus pares.

O último artigo da análise não está relacionado com formação de engenheiros e não possui relação direta com o ensino universitário. Mas os autores de A.15 trazem um contexto de formação de professores a partir da formação continuada, o que atende exatamente uma das lacunas mostradas até agora por meio dos resultados das análises. Por esse motivo ele não foi excluído do corpus de análise, e decidiu-se por ser importante trazer para a presente revisão sistemática tal abordagem.

Little (2015), autor de A.15 afirma que há muitas publicações acadêmicas sobre o modelo pedagógico Sala de Aula Invertida em países como os EUA, tanto da Educação Básica quanto no Ensino Superior. No entanto, também afirmam sobre a ausência da abordagem deste tema na formação do docente. O estudo possui por objetivo implementar um projeto de Sala de Aula Invertida para a formação de professores a fim de contribuir para a lacuna que também foi notada na presente revisão sistemática da literatura.

Os resultados da pesquisa de A.15 mostram que a proposta foi significativa, mas o autor alerta para novas aplicações na formação de professores, com amostras mais representativas, para que os dados possam ser amplamente analisados quanto as vantagens e desvantagens do projeto implementado. Além disso, é possível perceber, do mesmo modo que em A.11 e A.12, uma preocupação com o tempo do docente para o preparo das tarefas e materiais. Há, ainda, menção à questão da motivação docente para a proposição de metodologias diferentes, o que, segundo o autor pode estar relacionada à imensa carga de trabalho desses profissionais.

Outro fato destacado pelo autor de A.15 é a escassez de evidências que justifiquem a implementação da Sala de Aula Invertida, pois, como afirma em suas conclusões, o docente precisa justificar suas escolhas metodológicas e, para isso, necessita de evidências que comprovem os benefícios da metodologia em questão. Além de que, é imprescindível que haja material teórico que embase o trabalho com a sala invertida e que o professor tenha acesso à formação continuada, conforme já se observou em A.3, A.5, A.7, A.11, e A.12.

\footnotetext{
${ }^{6} \mathrm{O}$ estudo foi desenvolvido em uma Universidade específica, porém os autores não indicam qual e nem esclarecem se é pública ou privada e onde se localiza.
} 


\subsection{Análise quantitativa dos dados}

As 15 publicações analisadas anteriormente ilustram um cenário internacional da aplicação da Sala de Aula Invertida enquanto modelo pedagógico para a formação de engenheiros. Para além disto, foi possível realizar alguns levantamentos quantitativos a partir dos metadados coletados durante o procedimento da revisão.

Em princípio, foi possível identificar a atualidade do tema por meio da análise dos anos de publicação. Os autores Abeysekera e Dawson (2015) (A.1) afirmam que o termo Sala de Aula Invertida teve início em 2011, porém cresceu exponencialmente a partir de 2013. A Figura 1 traz um gráfico que demonstra a evolução deste tema a partir do número de artigos coletados nesta pesquisa.

Figura 1 - Número de artigos coletados por ano de publicação

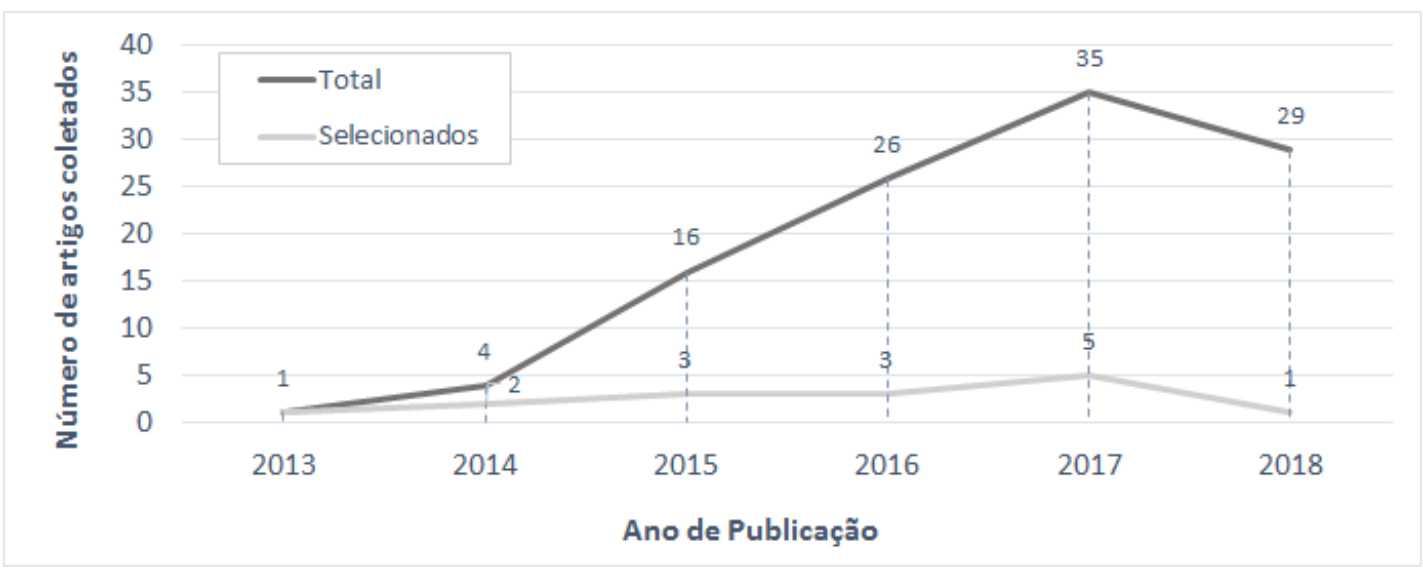

Fonte: Dados da Pesquisa.

A Figura 1 contempla os dados de todos os artigos coletados após o processo de filtragem (Total), bem como os 15 artigos selecionados para este estudo (Selecionados). Observa-se que somente a partir de 2013 foram encontrados artigos, concordando com a afirmação de Abeysekera e Dawson (2015) apontada anteriormente.

Nota-se que o ano de 2018 não seguiu a tendência de crescimento dos anos anteriores. Isto pode ser devido ao momento em que foi realizada a coleta (setembro/2018). Neste sentido os resultados oficiais de 2018 ainda são desconhecidos, sendo possível que se adicionem alguns documentos. Mesmo assim, a Figura 1 deixa evidente o crescimento do tema.

Outro levantamento realizado diz respeito aos autores que trabalham com o tema em questão e possíveis grupos de trabalho. Para tanto realizou-se a construção de um grafo de coautoria, selecionando autores e co-autores pertencentes ao grupo dos 15 artigos e identificando suas relações de coautoria considerando todos os 111 artigos (Figura 2). 


\section{Figura 2 - Grafo de coautoria}

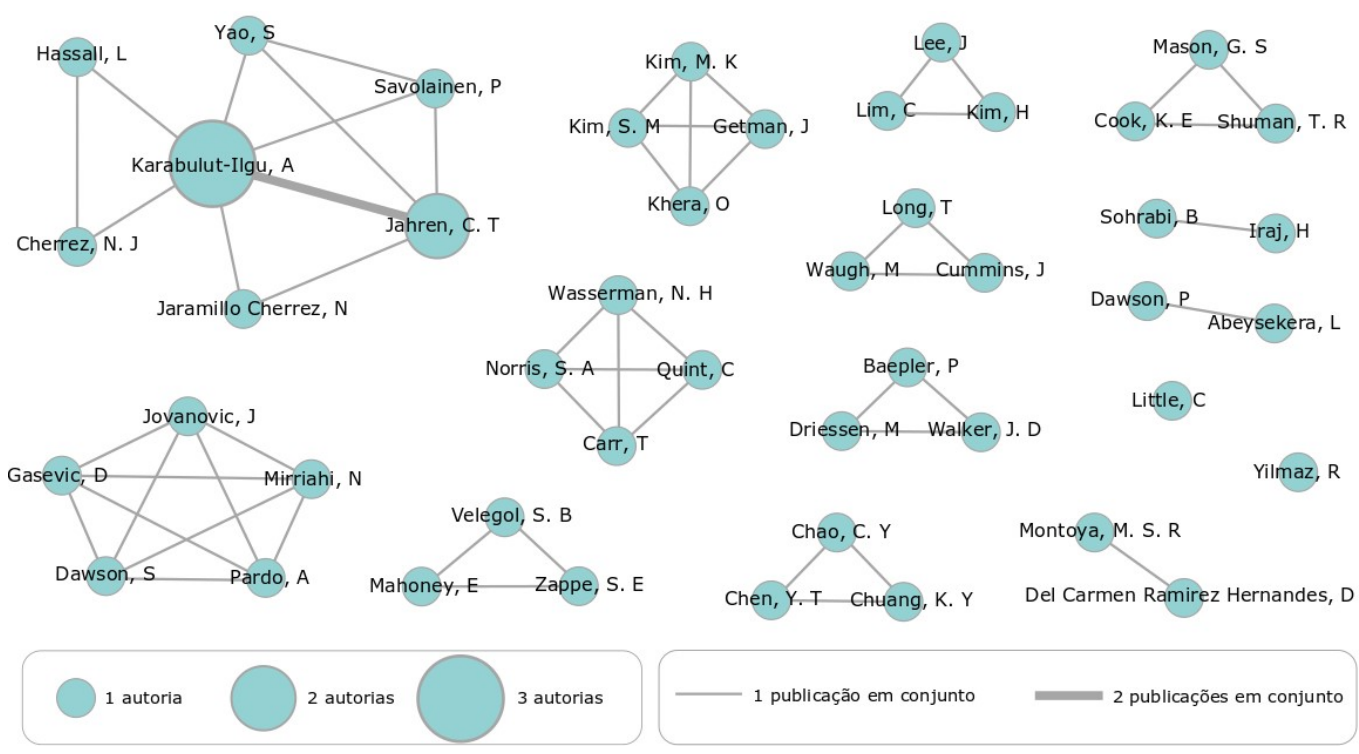

Fonte: Dados da Pesquisa.

Inicialmente nota-se, por meio da Figura 2, um isolamento dos grupos de pesquisa. Há diversos autores que atuam nesta linha, porém a maioria com apenas uma publicação, sugerindo que grupos de pesquisa ainda não estão estabelecidos. Nota-se este isolamento ao analisar que não há autores repetidos no Quadro 2, e que mesmo no grupo total de 111 artigos esses autores não aparecem novamente.

Em contrapartida, há um grupo centralizado nos autores Karabulut-Ilgu ${ }^{7}$ e $\mathrm{Jahren}^{8}$ (com três e dois artigos respectivamente). Nota-se que neste grupo houve três artigos publicados nesta temática e um deles na sexta posição da classificação InOrdinatio. Isto permite apontar para a importância desse grupo e sua consolidação ao redor dessa temática.

\section{CONSIDERAÇÕES FINAIS}

Diante dos dados apresentados, nota-se a viabilidade do uso do modelo pedagógico Sala de Aula Invertida no Ensino Superior, visando a formação de engenheiros. Dentre os benefícios, destaca-se a disponibilidade de tempo em sala de aula para interação professoraluno e aluno-aluno; a possibilidade de aprendizagem fora da sala de aula; a formação ativa do futuro engenheiro, contribuindo para o desenvolvimento do pensamento e da ação crítica.

Porém, a análise dos artigos se consolida e aponta para diversas lacunas ainda sem sinais de resoluções. Entre as lacunas destaca-se a ausência de material teórico que direcione e ofereça suporte para docentes e discentes quanto a Sala de Aula Invertida como modelo pedagógico; a dificuldade e falta de tempo para preparar os materiais a serem disponibilizados para os alunos antes da aula presencial; a falta de formação dos docentes para a utilização do modelo.

\footnotetext{
${ }^{7}$ Karabulut-Ilgu, Cherrez e Jahren, 2018; Karabulut-Ilgu, Yao, Savolainen, Jahren, C., 2018; Karabulut-Ilgu, Cherrez, Hassall, 2018.

${ }^{8}$ Karabulut-Ilgu, Cherrez, Jahren, 2018; Karabulut-Ilgu, Yao, Savolainen, Jahren, 2018.
} 
O levantamento quantitativo também mostrou que, embora as iniciativas sejam recentes e em constante crescimento nos últimos anos, a maioria dos autores identificados realizam pesquisas isoladas. Isto foi constatado por meio da identificação de apenas um grupo de pesquisa com destaque nesse tema. Sugerindo assim uma maior consolidação do tema em questão.

Diante das lacunas identificadas, verifica-se um trabalho preocupado com a formação dos docentes, pois conhecendo os benefícios e vantagens da Sala de Aula Invertida, se faz necessário sua implantação no processo de ensino. Conclui-se, portanto, que há a necessidade de novas intervenções na formação dos docentes que viabilizem suporte e direcionamento para a prática do professor na Sala de Aula Invertida, e antes disso, são necessárias novas pesquisas que construam caminhos a serem seguidos em formato teórico para que possam servir de base para essas intervenções e futuras práticas. 


\section{REFERÊNCIAS}

ABEYSEKERA, L.; DAWSON, P. Motivation and cognitive load in the flipped classroom: definition, rationale and a call for research. Higher education research \& development, v. 34, n. 1, p. 1-14, 2015.

BAEPLER, P.; WALKER, J. D.; DRIESSEN, M. It's not about seat time: Blending, flipping, and efficiency in active learning classrooms. Computers \& Education, v. 78, p. 227-236, 2014.

BORGES, T. S.; ALENCAR, G. Metodologias ativas na promoção da formação crítica do estudante: o uso das metodologias ativas como recurso didático na formação crítica do estudante do ensino superior. Cairu em revista, v. 3, n. 4, 2014.

CHAO, C.Y.; CHEN, Y. T.; CHUANG, K. Y. Exploring students' learning attitude and achievement in flipped learning supported computer aided design curriculum: A study in high school engineering education. Computer Applications in Engineering Education, v. 23, n. 4, p. 514-526, 2015.

JOVANOVIĆ, J. et al. Learning analytics to unveil learning strategies in a flipped classroom. The Internet and Higher Education, v. 33, n. 4, p. 74-85, 2017.

KARABULUT-ILGU, A.; JARAMILLO CHERREZ, N.; JAHREN, C. T. A systematic review of research on the flipped learning method in engineering education. British Journal of Educational Technology, v. 49, n. 3, p. 398-411, 2018.

KIM, M. K. et al. The experience of three flipped classrooms in an urban university: an exploration of design principles. The Internet and Higher Education, v. 22, p. 37-50, 2014.

LEE, J.; LIM, C.; KIM, H. Development of an instructional design model for flipped learning in higher education. Educational Technology Research and Development, v. 65, n. 2, p. 427453, 2017.

LITTLE, C. The flipped classroom in further education: literature review and case study. Research in Post-Compulsory Education, Taylor \& Francis, v. 20, n. 3, p. 265-279, 2015.

LONG, T.; CUMMINS, J.; WAUGH, M. Use of the flipped classroom instructional model in higher education: instructors' perspectives. Journal of Computing in Higher Education, v. 29, n. 2, p. 179-200, 2017.

MASON, G. S.; SHUMAN, T. R.; COOK, K. E. Comparing the effectiveness of an inverted classroom to a traditional classroom in an upper-division engineering course. IEEE Transactions on Education, v. 56, n. 4, p. 430-435, 2013.

PAGANI, R. N.; KOVALESKI, J. L.; RESENDE, L. M. Methodi ordinatio: a proposed methodology to select and rank relevant scientific papers encompassing the impact factor, number of citation, and year of publication. Scientometrics, v. 105, n. 3, p. 2109-2135, 2015.

PINTRICH, P. R.; GROOT, E. V. De. Motivational and self-regulated learning components of classroom academic performance. Journal of educational psychology, v. 82, n. 1, p. 33-40, 1990.

RAMÍREZ-MONTOYA, M. S.; HERNÁNDEZ, D. C. R. Inverted learning environments with technology, innovation and flexibility: Student experiences and meanings. Journal of Information Technology Research (JITR), v. 9, n. 1, p. 18-33, 2016. 
SCOPUS PREVIEW. <https://www.scopus.com/home.uri>, 2019.

SOHRABI, B.; IRAJ, H. Implementing flipped classroom using digital media: A comparison of two demographically different groups perceptions. Computers in Human Behavior, v. 60, p. 514-524, 2016.

VALENTE, J. A. A sala de aula invertida e a possibilidade do ensino personalizado: uma experiência com a graduação em midialogia. In: BACICH,, L. MORAN, J. (Orgs.). Metodologias ativas para uma educação inovadora: uma abordagem teórico-prática. Porto Alegre: Penso, 2018. p. 26-44.

VELEGOL, S. B.; ZAPPE, S. E; MAHONEY, E. M. I. L. Y. The evolution of a flipped classroom: Evidence-based recommendations. Advances in Engineering Education, v. 4, n. 3, p. 1-37, 2015.

WASSERMAN, N. H. et al. Exploring flipped classroom instruction in calculus iii. International Journal of Science and Mathematics Education, v. 15, n. 3, p. 545-568, 2017.

YILMAZ, R. Exploring the role of e-learning readiness on student satisfaction and motivation in flipped classroom. Computers in Human Behavior, v. 70, p. 251-260, 2017. 